\title{
III.
}

\section{Ein Fall von Knochenverschmelzungen im Bereiche des Fussskelettes.}

\author{
Von Dr, Alexander Brenner, \\ Prosector an Wiener anatomischen Institute.
}

(Hierza Taf. II.)

Coalitionen einzelner Fusswurzelknochen sind wohl schon in ziemlicher Menge bekannt; namentlich sind es die Keilbeine, welche nicht selten unter einander verschmolzen; in der Regel aber bleibt an der Verwachsungsstelle eine deutliche Rinne zurück, welche auf den ersten Blick die Horkunft des Doppelknochens erkennen lässt. In einem Falle, den W. Cruber in diesem Archive, Bd. 49, beschreibt, schliesst sich an eine solehe Verwachsung noch eine analoge Bildung im Bereiche des Mittelfusses an; es trägt das aus dem zweiten und dritten Keilbein zusammengesetate Knochenstück vorne nur einen besonders mächtigen Metatarsus, „der den 2. und 3. Metatarsus substituirt", es sind also nur 4 Mittelfussknochen und nur 4 Zehen.

Der Fall, den ich hier zu beschreiben gedenke, ist auch dadareh charakterisirt, dass die vordere Reihe der Fusswurzelknochen um einen Knochen, und zwar um ein Keilbein zu wenig zählt; doch finden sich 5 Mittelfussknochen und 5 Zehen: Nirgends besteht eine deutliche Markirung jener Knochon, die unter einander verschmolzen sind oder sein könnten.

Bei der genaneren Betrachting der Fusswurzelknochen ergiebt sich, dass das Sprung- und Fersenbein, sowie auch das innere oder erste Keilbein in Form und Grösse der Norm entsprechen.

Das zweite Keilbein erscheint zunächst etwas breiter als gewöhnlich, seine innere dem Entocuneiforme zugewendete Hälfte trägt alle Merkmale eines zweiten Keilbeines. Die äussere dem Würfelbeine zugekehrte Seite ist der Articulation mit demselben entzprechend etwas abweichend gestaltet. Vor dem kleinen ausspringenden Winkel bei a (Fig. 1) beginnt eine plantarwärts verlaufende Rinne.

Vor derselben befindet sich an der vorderen Grenzkante dieser Seite des Keilbeines eine kleine halblinsenförmige Gelenksfacette, welche mit der 


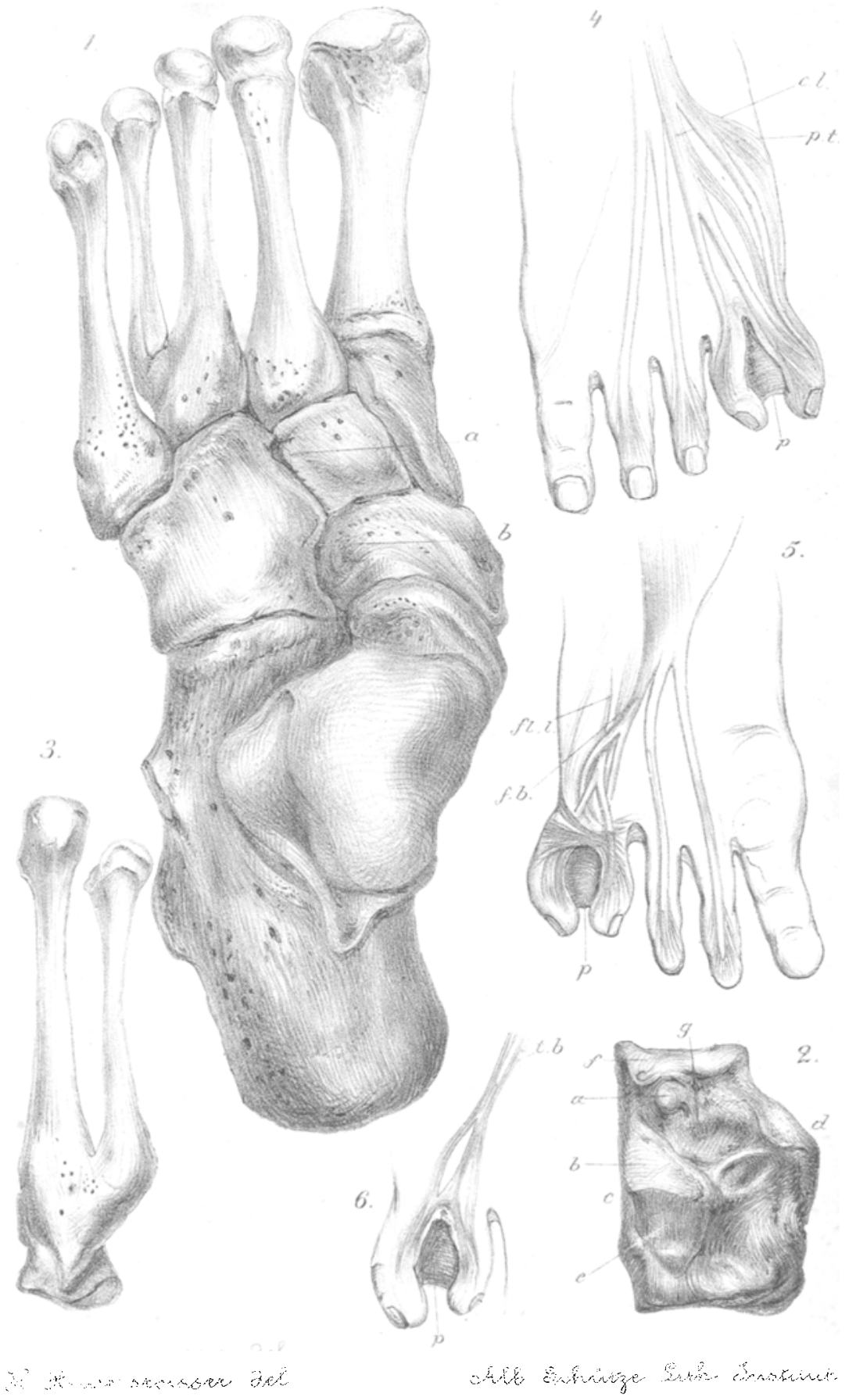


gleichgestalteten Fläche des Cuboideums bei a Fig. 2 articulirt. Hinter der Rinne beginnt eine ungefähr dreieckige Gelenksfläche, deren Basis die hintere Grenzkante des Keilbeines bildet; sie articulirt mit einer gleichgestalteten Facette des Cuboideums bei b Fig. 2.

Diese, wie die vorhin erwähnte Gelenksfläche, finden sich normaler Weise an der Aussenfläche des dritten Keilbeines. In der Mitte dieser Seite des zweiten Keilbeines finden sich mehrere rautenförmig geordnete Knochenhöckerchen, die auch an normalen Keilbeinen, aber nicht so stark entwickelt, zu sehen sind.

Das Würfelbein zeigt in Form und Grösse die meisten Abweichungen von einem normalen.

Die Grössendifferenz bezieht sich hauptsächlich auf die innere Hälfte des Knochens; es beträgt an der Innenseite der Abstand des vorderen von dem hinteren Rande $3,5 \mathrm{~cm}$ gegen $2,7 \mathrm{~cm}$ eines normalen Cuboideum von einem gleich stark gebanten Fusse, an der Aussenseite $2,0 \mathrm{~cm}$ gegen $1,8 \mathrm{~cm}$ desselben Cuboideums.

Auch der grösste Abstand des medialen vom lateralen Rande des Knoehens ist vergrössert; er beträgt $3,4 \mathrm{~cm}$, während er an dem normalen Würfelbeine nur 2,7 cm misst; ebenso verhält es sich mit dem Dickendurchmesser in der medialen Hälfte, der $2,5 \mathrm{~cm}$ gegenüber $2,2 \mathrm{~cm}$ des verglichenen normalen Cuboideums beträgt.

Ohne Zweifel ist die innere Hälfte des Würfelbeines, wie sich schon bei blossem Ansehen desselben ergiebt, grösser als an einem normalen Fusse.

Aber auch die Form differirt einigermaassen; so fällt bei Betrachtung der dorsalen Seite des Knoebens der steile Uebergang von der vorderen lateralen zur medialen Articulationsfïche mit den Metatarsalknochen auf, Fig. 1.

Die mediale Begrenzungslinie springt etwas hinter der Mitte als Sporn vor, hinter demselben zieht sie in einem concaven Bogen zur hinteren Gelenkfläche des Knochens, vor demselben in gerader nach aussen abweichender Richtung zur vorderen Gelenkfläche; bevor sie diese erreicht, bildet sie noch einen kleinen ausspringenden Winkel, welcher gegen die oben bezeichnete Rinne des zweiten Keilbeines (vor a Fig. 1) gerichtet ist. An der medialen dem zweiten Keilbeine zugekehrten Seite (Fig. 2) des Würfelbeines entspricht dem Sporne eine die ganze Seite plantarwärts (von $\mathrm{c}-\mathrm{d}$ ) durchziehende Ieiste, welche dorsalwärts zwei Gelenkflächen $b$ und e von einander scheidet, plantarwärts in den Knochenwulst der unteren Seite des Würfelbeines übergeht (bei d).

An der Grenze dieser Seite gegen die Tarsometatarsalgelenksflächen des Cuboideums ist eine beilänfig $4 \mathrm{~mm}$ hohe rechteckige Gelenksfacette zur Articulation mit dem zweiten Metatarsus (bei f Fig. 2).

Hinter derselben die kleine Facette bei a, welehe mit der analogen $\mathrm{Fa}-$ cette des zweiten Keilbeines articulirt.

Ausserdem finden sich in dieser vor der Leiste cd gelegenen IIälfte dieser Seite noch kleine rosettenförmige Knochenböckerchen bei $\mathrm{g}$, ähnlich den oben beim zweiten Keilbein beschriebenell. An der Stelle, wo sich 
hinter der Leiste c d die zackige Gelenkfläcbe c findet, ist an normalen Würfelbeinen entweder ein kleiner Höcker oder eine ganz kleine Gelenksfacette, für eine Verbindung mit dem Naviculare.

Dieses ist in Form und Grösse eigentlich normal; an seiner vorderen Seite trägt es zwei grosse Gelenksfacetten für die beiden Keilbeine, und an diese schliesst sich lateralwärts eine ganz kleine convexe dritte Facette an, welche durch eine allerdings nur schwach angedentete Leiste (dorsalwärts durch den Vorsprung bei b Fig. 1) in zwei Hälften zerfällt, von denen die mediale der normalen Articulationsfläche eines Naviculare mit dem dritten Keilbeine, aber in verkleinertem Maassstabe, die laterale der Verbindung mit dem Würfelbeine an einem normalen Skelette entspricht. Beide diese Hälften bilden hier eine dreieckige Gelenksfacette, welche mit der gleichen des Cuboideum bei c Fig. 2 articulirt.

Entsprechend der Zahl der Gelenksflächon, welche in diesem Falle von. den Fusswurzelknochen zur Articulation mit den Mittelfussknochen dargehoten werden, sollte es ejgentlich nur 4 Metatarsi geben. In der That articuliren auch nur 4 in der Lisfranc'schen Linie: der erste Metatarsus mit dem ersten Keilbeine, der zweite mit dem zweiten, der dritte mit der medialen, der fünfte mit der lateralen Facette des Guboideum. Der vierte Metatarsus ist aus der Reihe der übrigen nach vorne verschoben in das Interstitium interosseum hinein und da der dritte und fünfte Metatarsus basalwärts aneinandersehliessen (Fig. 1), ist der vierte ausser Stand gesetzt mit dem Tarsus zu articuliren. Es ist die Basis des vierten Metatarsus an einen von der Basis des dritten lateral und abwärts gerichteten konischen Knochenfortsate (Fig. 3) aufgesetzt und Iurch einen Knochenspalt, der in Fig. 1 deutlich dargestellt ist, seine Grenze markirt. Dadurch ist seine Basis schon um $2 \mathrm{~cm}$ von dem Cuboideum entfernt gehalten, ausserdem ist der ganze Knochen im Wachsthum bedeutend zurückgeblieben; er ist um die Hälfte dünner als seine Nachbarn, er ist leicht gekrümmt und ist um $2 \mathrm{~cm}$ kürzer als die anderen. Gleichzeitig ist sein Capitulum noch durch Epiphysenknorpel mit der Diaphyse verbunden. Ihas Capitulum aber batte sich in die Reihe der übrigen Metatarsi eingestellt und es war äusserlich dieses merkwürdige Verhalten des vierten Metatarsus nur angedeutet durch eine dreieckige Grube, deren tiefste Stelle nach rückwärts lag zwischen dem dritten und fünften Mittelfussknochen.

Die Phalangen der 3 ersten Zehen waren normal; die Grundphalangen der vierten und der fünften Zehe aber waren an ihrer Basis miteinander knöchern verbunden und von da aus divergirten die beiden nach vorn. Die Gelenksflächen tür den vierten und fünften Metatarsus waren vollständig gesondert, ebenso anch für jedes Gelenk eine selbständige Gelenkskapsel vorhanden. Auf den divergirenden Grundphalangen der vierten und fünften Zehe sassen dann die zweiten Phalangen unter stumpfem Winkel in der Weise auf, dass die Zehenspitzen einander zusahen und gleichzeitig die Zehenrücken plantarwärts etwas rotirt waren, wodurch ein lyraförmiger Raum zwischen vierter und fünfter Zehe zu Stande kam. 
Es entsteht nun die Frage, ist ein drittes Keilbein überhaupt angelegt gewesen und ist es mit einem Nachbarknochen verschmolzen, oder fehlte die Anlage eines dritten Keilbeines gänzlich und haben sich dafür die Nachbarknochen, das zweite Keilbein und das Würfelbein um so intensiver entwickelt? Mit Rücksicht auf die Verhältnisse im Bereiche des Mittelfusses und der Zehen erscheint es nun naheliegend, auch in der Fusswurzel Coalition anzunehmen, zumal sich hier das Cuboideum als Knochen darbietet, der an und für sich schon durch seine Grösse und Form mehr darzustellen scheint als ein gewöhnliches Würfelbein; es articulirt ferner dieses Cuboideum durch seine stärker entwickelte mediale Hälfte mit dem Naviculare durch zwei durch eine Leiste geschiedene Gelenksfacetten (bei e Fig. 2) annähernd wie ein normales Ektocuneiforme, und schliesslich trägt es drei Mittelfussknochen. Wenn man nun annimmt, dass das dritte Keilbein durch Verschmelzung seines Knochenkernes mit dem des Würfelbeines in dem letzteren aufging, würde es nahe liegen ein gleiches Verhältniss auch in den Metatarsis zu erwarten, so wie in Gruber's Fall (l. c.), wo der Coalition zwischen zweitem und drittem Keilbeine eine Verschmelzung des zweiten und dritten Metatarsus zu einem besonders mächtigen Knochen entspricht; dem ist in unserem Falle nicht so; es ist der dritte Metatarsus nicht im vierten aufgegangen, wie das dritte Keilbein im Würfelbeine, es ist vielmehr der dritte Metatarsus kräftig entwickelt und der vierte, der mit der medialen Gelenksfläche des Cuboideum articuliren sollte, ist atrophisch, ist aus seiner Verbindung mit der Fusswurzel verrückt und ein Appendix des dritten geworden; man muss also annehmen, dass der Knochenkern des dritten Keilbeines schon in sehr früher Zeit mit dem des Würfelbeines verwachsen ist, und sich im Rahmen des Würfelbeines kräftig weiter entwickelte auf Kosten der medialen Hälfte des letzteren; und in Folge dieser Verhältnisse im Cuboideum hatte schliesslich der vierte Metatarsus keinen Articulationsknochen nach rückwärts und wurde durch seine kräftig wachsenden Nachbarn von dem Cuboideum abgedrängt.

Auffällig ist auch ein gewisses Fortschreiten der Coalition von innen nach aussen und vorne; es ist das dritte Keilbein mit dem Würfelbeine, der vierte Metatarsus mit dem dritten und 


\section{die Grundphalange der fünften Zehe mit der der vierten Zehe verwachsen.}

Diesen angebornen Knochenverhältnissen entsprechend waren natürlich auch die Weichtheile, die Sehnen und Muskeln angeordnet.

Aeusserlich war an dem Fusse zunächst nur die Syndaktylie zwischen den beiden letaten Zehen aufgefallen und der lyraförmige Raum, der durch die beiden eingeschlossen war. In diesem Raume war von einer zur anderen Zehe eine derbe Bindegewebsmembran ausgespannt ( $p$ in Fig. 4-6), die in das Periost der Phalangen äberging und nach vorn bis zu den Nagelphalangen reichte.

Die Strecksehnen beider Zehen gehen aus der Theilung einer gemeinsamen Sehne des Extensor communis longus hervor (Fig. 4) und übergehen an den Basen der Grundphalangen in die Streckaponeurosen. Von dor Sehne des M. peroneus tertius bündelt sich, wie ja das häufig ler Fall ist, ein kleines Faseikel los, welches mit einem von der geneinsamen Streeksehne der beiden letzten Zehen früb abgetrennten Sehnenbündel vereint als zweite Sebne zur Streckaponeurose der kleinen Zehe zieht. Durcb die oben erwähnte Rotation beider Zehen um ihre Längsaxe kommen die Streckaponeurosen natülich nicht dorsal sondern medial und lateral zu liegen.

In der Sohle spaltet sich dio für beide Zehen gemeinsane Sebne des Flexor brevis (Fig. 5 u. 6) vor den Motatarsophalangealgelenken in zwei dünne Sehnen; die laterale zieht zur Basis der zweiten Phalanx der kluinen Zehe, die mediale theilt sich nach kurzem Vertaufe wieder in zwei Bändel, von denen das innere direct atur Nagelphalange der vierten Zehe verfolgt, werden kamn, während das änssere sich zunïchst mit der Sehne der kleinen Zehe verbindet, dann aber stumpfwinklig ron derselben abbiegt und an Aussenrande der vierten Zehe zur Basis der zwciten Phalange zicht.

Durch die Spaltung dor gemeinsamen Sehno des Flexor brevis in zwoj Sehnen und deren neuerliche Verbindung untereinander wird eine Läeke gebililet, dureh welche die Sehne des M. flexor. com. longus durchtritt; erst nach ihrem Durchtritt theilt letztere sich in zwei Sehnen, wolche nun, die Sebnenbündel des M. flexor brevis bedeckerıd, zu den Nagelphalangen beider Zehen ziehen.

Im Bereiche der beiden verwachsenen Grundphalangen hatten beide Sehnen eine gemeinsame Scheide mit deutlichen gekreuzten Fasern.

Die letzten zwei Lumbricalrauskeln feh]ten, ebenso waren die Interossei im dritten und vierten Intermetatarsalraume nur sehr wenig entwickelt. Die Interossei des vierten Netatarsalraumes inserirten sich an einem kleinen zwischen den beiden selbständigen Metatarsophalangealgelenken gelegenen und mit den Gelenkskapseln verwachsenen Sesambeinchen.

Die Haut deckte dorsal- und plantarwärts die verbildeten Theile bis zur Nagelpbalange und liess niebts auffälig erscheinen als die Syndaktylie der zwei letzten Zehen. 


\title{
Erklärung der Abbildungen. Tafel $\mathrm{II}$.
}

Fig. 1. Ansicht des Fussskeletes von oben.

Fig. 2. Mediale, dem Keilbeine und dem Naviculare zugewendete Seite des Würfelbeines.

Fig. 3. a Dritter, b vierter Metatarsusknochen in der Ansicht von unten.

Fig. 4. Sehnen am Fussrücken. pt Peroneus tertius. el Extensor com. longus. p Sehnenplatte zwischen den beiden Zehen.

Fig.5 u. 6. Sehmen an der Plantarseite. $f$ b Sehne des Flexor brevis. f 1 Sehne des Flexor longus. p Sehnenplatte zwischen beiden Zehen.

\section{IV.}

\section{Abu's-'Salt (gest. 1134) und seine Simplicia,}

\author{
ein Beitrag zur Heilmittellehre der Araber, \\ von M. Steinschneider in Berlin.
}

Zu den Vorläufern und Quellen ibn Beithar's in Spanien gehört abu's-Salt, welchen Ernst H. F. Meyer (Gesch. d. Botanik III, 345) nur im Anhange des Kapitels, nach Hagi Khalfa, als Verf. einer Schrift über einfache Heilmittel erwähnt, ohne auf jrgend eine Quelle $z$ u verweisen. Nach Leclerc (1. citando II, 74) ist er ungefähr 20 mal von IB. angeführt. Ich habe diese Stellen nicht gesammelt, nur einige in Sontheimers Uebersetzung meist uncorrecte Citate notirt, nämlich: Ebn Abi S. II, 66, Abu'l Sult 361, 403, Amih Ben Abi Solt II, 58, vgl. II. 37; in den "Biographien“ Sontheimers hat er keinen Platz gefunden.

\section{$\S 1$.}

Der correcte Namen ist: [ibn] abu's-Salt Omeïje b. Abdi'l-Aziz. Er war als Arzt bekannt, aber auch als Mathematiker und Dichter. Artikel über ihn findet man in dem unedirten Gelehrten-Lexicon des Vezir's el-Kifti, in der Geschichte der Aerzte O'seibia's - deren baldige Ausgabe durch Prof. Aug. Müller (jetzt in Königsberg) mich veranlasst, einige Details unerörtert zu lassen, - und im biographischen Wörterb. des ibn 